\title{
OCCUPATION TIMES FOR MARKOV-MODULATED BROWNIAN MOTION
}

\author{
LOTHAR BREUER, ${ }^{*}$ University of Kent
}

\begin{abstract}
In this paper we determine the distributions of occupation times of a Markov-modulated Brownian motion (MMBM) in separate intervals before a first passage time or an exit from an interval. We derive the distributions in terms of their Laplace transforms, and we also distinguish between occupation times in different phases. For MMBMs with strictly positive variation parameters, we further propose scale functions.
\end{abstract}

Keywords: Markov-modulated Brownian motion; occupation time; scale function; Markov additive process

2010 Mathematics Subject Classification: Primary 60J25

Secondary 60G51; 60J55

\section{Introduction}

Let $g=\left(J_{t}: t \geq 0\right)$ denote an irreducible Markov process with finite state space $E=$

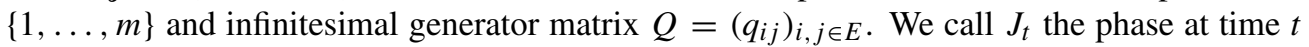
and $\mathcal{g}$ the phase process. Choosing parameters $\mu_{i} \in \mathbb{R}$ and $\sigma_{i} \geq 0$ for all $i \in E$, we define the level process $\mathcal{X}=\left(X_{t}: t \geq 0\right)$ by

$$
X_{t}=X_{0}+\int_{0}^{t} \mu_{J_{s}} \mathrm{~d} s+\int_{0}^{t} \sigma_{J_{s}} \mathrm{~d} W_{s}
$$

for all $t \geq 0$, where $\mathcal{W}=\left(W_{t}: t \geq 0\right)$ denotes a standard Wiener process that is independent of $\mathcal{g}$. Then $(\mathcal{X}, \mathcal{g})$ is called a Markov-modulated Brownian motion (MMBM). An MMBM is a Markov additive process (MAP; see [2, Chapter XI]) without jumps.

MMBMs have proved to be powerful tools in stochastic modelling, with applications in queueing theory, insurance, and finance. This is even more apparent after one considers the fact that exit problems for MAPs with phase-type jumps can be reduced to an analysis of MMBMs by standard transformation techniques (see, e.g. [6] and [13]). The class of MAPs with phase-type jumps is dense within all MAPs; see Proposition 1 of [3]. Thus, we deal with occupation times for a dense subset of MAPs.

Some results for MMBMs go back to the 1990s, with Rogers [15] investigating WienerHopf factorisation and stationary distributions for the case that $\sigma_{i}=\varepsilon$ is independent of the phase process. Around the same time, Asmussen [1] determined the hitting probabilities and, based on these, expressions for the stationary distributions. More recent results are given in [7] and [10], where MMBMs with two reflecting barriers are analysed. Some properties of scale functions for MMBMs are derived in [12].

Occupation times for the phase process before a one- or two-sided exit can be determined via the results in [6] and [13]. This will be discussed in Section 2. As an afterthought to this,

Received 5 July 2011; revision received 22 November 2011.

* Postal address: Institute of Mathematics and Statistics, University of Kent, Canterbury CT2 7NF, UK.

Email address: 1.breuer@kent.ac.uk 
we shall propose a definition of scale functions for MMBMs with strictly positive variation parameters, i.e. $\sigma_{i}>0$ for all $i \in E$, in Section 3. The more challenging part will be the determination of occupation time distributions for the combined level and phase process. This is the content of Section 4, which deals with the case of only two intervals. The generalisation to more than two intervals is then described in the last section. Appendix A contains some lemmata that are used in the proofs of the main results.

\section{Preliminaries: occupation times of the phase process}

\subsection{Occupation times before a first passage}

Define the first passage times $\tau(x):=\inf \left\{t \geq 0: X_{t}>x\right\}$ for all $x \geq 0$, and assume that $X_{0}=0$. We are interested in the occupation times $\zeta_{j}(x):=\int_{0}^{\tau(x)} \mathbf{1}_{\left\{J_{t}=j\right\}} \mathrm{d} t$ in a phase $j \in E$ before the first passage over the level $x \geq 0$. We collect these occupation times in the column vector $\zeta(x):=\left(\zeta_{j}(x): j \in E\right)$. Consider an $E$-dimensional row vector $\boldsymbol{r}=\left(r_{i}: i \in E\right)$ with nonnegative entries $r_{i} \geq 0$ for all $i \in E$. Define

$$
\mathrm{E}_{i j}\left(\exp \left[-\int_{0}^{\tau(x)}{ } r_{J_{s}} \mathrm{~d} s\right]\right):=\mathrm{E}\left(\exp \left[-\int_{0}^{\tau(x)} r_{J_{s}} \mathrm{~d} s\right] ; J_{\tau(x)}=j \mid J_{0}=i, X_{0}=0\right)
$$

for $i, j \in E$ and $\mathrm{E}\left(\exp \left[-\int_{0}^{\tau(x)} r_{J_{s}} \mathrm{~d} s\right]\right)$ as the $(E \times E)$-matrix with these entries. Noting that $\exp \left[-\int_{0}^{\tau(x)} r_{J_{s}} \mathrm{~d} s\right]=\mathrm{e}^{-\boldsymbol{r} \zeta(x)}$ we see that the matrix $\mathrm{E}\left(\exp \left[-\int_{0}^{\tau(x)} r_{J_{S}} \mathrm{~d} s\right]\right)$ contains the joint Laplace transforms of the occupation times $\zeta_{j}(x)$.

In order to determine $\mathrm{E}\left(\exp \left[-\int_{0}^{\tau(x)} r_{J_{s}} \mathrm{~d} s\right]\right)$, we shall distinguish the phases by the subspaces

$$
E_{a}:=\left\{i \in E: \sigma_{i}>0 \text { or } \mu_{i}>0\right\} \text { and } E_{d}:=E \backslash E_{a},
$$

where phases in $E_{a}$ and $E_{d}$ are respectively called ascending and descending. The same arguments as in [5, Section 3] yield

$$
\mathrm{E}_{(d, d)}\left(\exp \left[-\int_{0}^{\tau(x)}{ }^{2} r_{J_{s}} \mathrm{~d} s\right]\right)=\mathrm{E}_{(a, d)}\left(\exp \left[-\int_{0}^{\tau(x)} r_{J_{s}} \mathrm{~d} s\right]\right)=\mathbf{0}
$$

as well as

$$
\begin{aligned}
& \mathrm{E}_{(d, a)}\left(\exp \left[-\int_{0}^{\tau(x)} r_{J_{s}} \mathrm{~d} s\right]\right)=A(\boldsymbol{r}) \mathrm{e}^{U(\boldsymbol{r}) x} \\
& \text { and } \mathrm{E}_{(a, a)}\left(\exp \left[-\int_{0}^{\tau(x)} r_{J_{s}} \mathrm{~d} s\right]\right)=\mathrm{e}^{U(\boldsymbol{r}) x},
\end{aligned}
$$

where the matrices $A(\boldsymbol{r})$ and $U(\boldsymbol{r})$ can be computed as follows. For arguments $\beta \geq 0$, define the functions $\phi_{i}(\beta):=\beta / \mu_{i}$ for $i \in E_{a}$ and $\sigma_{i}=0$, as well as

and

$$
\phi_{i}(\beta)=\frac{1}{\sigma_{i}} \sqrt{2 \beta+\frac{\mu_{i}^{2}}{\sigma_{i}^{2}}}-\frac{\mu_{i}}{\sigma_{i}^{2}}
$$

$$
\phi_{i}^{*}(\beta)=\frac{1}{\sigma_{i}} \sqrt{2 \beta+\frac{\mu_{i}^{2}}{\sigma_{i}^{2}}}+\frac{\mu_{i}}{\sigma_{i}^{2}}
$$

for $i \in E_{a}$ and $\sigma_{i}>0$. The iteration to determine $A(\boldsymbol{r})$ and $U(\boldsymbol{r})$ is slightly changed from [6, Section 2.2]. That is, we obtain $(A(\boldsymbol{r}), U(\boldsymbol{r}))=\lim _{n \rightarrow \infty}\left(A_{n}, U_{n}\right)$ for initial values $A_{0}:=\mathbf{0}$, 
$U_{0}:=-\operatorname{diag}\left(\phi_{i}\left(q_{i}+r_{i}\right)\right)_{i \in E_{a}}$, and iterations

$$
e_{i}^{\prime} U_{n+1}=-\frac{q_{i}+r_{i}}{\mu_{i}} e_{i}^{\prime}+\frac{1}{\mu_{i}} \sum_{j \in E, j \neq i} q_{i j} e_{j}^{\prime}\left(\begin{array}{c}
I_{a} \\
A_{n}
\end{array}\right)
$$

for $i \in E_{a}$ and $\sigma_{i}=0$,

$$
e_{i}^{\prime} U_{n+1}=-\phi_{i}\left(q_{i}+r_{i}\right) e_{i}^{\prime}+\frac{2}{\sigma_{i}^{2}} \sum_{j \in E, j \neq i} q_{i j} e_{j}^{\prime}\left(\begin{array}{c}
I_{a} \\
A_{n}
\end{array}\right)\left(\phi_{i}^{*}\left(q_{i}+r_{i}\right) I-U_{n}\right)^{-1}
$$

for $\sigma_{i}>0$, and

$$
e_{i}^{\prime} A_{n+1}=\sum_{j \in E, j \neq i} q_{i j} e_{j}^{\prime}\left(\begin{array}{c}
I_{a} \\
A_{n}
\end{array}\right)\left(\left(q_{i}+r_{i}\right) I+\mu_{i} U_{n}\right)^{-1}
$$

for $i \in E_{d}$. Here $e_{i}^{\prime}$ denotes the $i$ th canonical row base vector, $q_{i}:=-q_{i i}$ for all $i \in E$, and $I_{a}$ is the identity matrix on $E_{a}$. The case $\boldsymbol{r}=\mathbf{0}$ has been analysed earlier in [1].

Remark 1. Let us add an absorbing phase, say $\Delta$, to the phase space $E$ to obtain $E^{\prime}=E \cup\{\Delta\}$. Define an MMBM $\left(X^{\prime}, g^{\prime}\right)$ on $E^{\prime}$ as follows. The generator matrix $Q^{\prime}$ of $g^{\prime}$ shall be given by

$$
q_{i j}^{\prime}:= \begin{cases}q_{i j}, & i, j \in E, j \neq i \\ q_{i i}-r_{i}, & j=i \in E, \\ r_{i}, & i \in E, j=\Delta, \\ 0, & i=\Delta, j \in E^{\prime}\end{cases}
$$

Furthermore, let

$$
\left(\mu_{i}^{\prime}, \sigma_{i}^{\prime}\right):= \begin{cases}\left(\mu_{i}, \sigma_{i}\right), & i \in E \\ (0,0), & i=\Delta\end{cases}
$$

which means that the phase $\Delta$ governs the zero process. Let $\tau_{\Delta}:=\min \left\{t \geq 0: J_{t}^{\prime}=\Delta\right\}$ denote the time until absorption in $\Delta$, and let $\tau^{\prime}(x):=\inf \left\{t \geq 0: X_{t}^{\prime}>x\right\}$ be the first passage time of $\chi^{\prime}$ over the level $x \geq 0$. Then

$$
\mathrm{E}_{i j}\left(\exp \left[-\int_{0}^{\tau(x)} r_{J_{s}} \mathrm{~d} s\right]\right)=\mathrm{P}\left(\tau^{\prime}(x)<\tau_{\Delta}, J_{\tau^{\prime}(x)}^{\prime}=j \mid J_{0}^{\prime}=i, X_{0}^{\prime}=0\right)
$$

for $i, j \in E^{\prime} \backslash\{\Delta\}$, i.e. the generalised Laplace transforms of the first passage times $\tau(x)$ can be seen as transition probabilities among the transient phases $i, j \in E^{\prime} \backslash\{\Delta\}$ for the phase process $g^{\prime}$ which terminates at a constant rate $r_{i}$ during $\left\{t \geq 0: J_{t}^{\prime}=i\right\}$. Thus, we call $\boldsymbol{r}$ the exit rate vector.

From this perspective, a phase-type distribution with parameters $(\alpha, T)$ on a phase space $E$ can be translated as follows. Let $\eta:=-T \mathbf{1}$ denote the exit vector, and let $t_{i j}$ denote the entries of $T$. Consider a random variable $Z \sim \operatorname{PH}(\alpha, T)$. Setting $r_{i}:=\eta_{i}, q_{i j}:=t_{i j}$ for $i \neq j \in E$, and $\left(\mu_{i}, \sigma_{i}\right)=(1,0)$ for all $i \in E$ yields $U(\boldsymbol{r})=T$, and, thus,

$$
\mathrm{P}(Z>x)=\mathrm{P}_{\alpha}\left(\tau^{\prime}(x)<\tau_{\Delta} \mid X_{0}^{\prime}=0\right)=\alpha \mathrm{e}^{T x} \mathbf{1},
$$

where $\mathrm{P}_{\alpha}$ denotes the conditional probability given that $\mathrm{P}\left(J_{0}^{\prime}=i\right)=\alpha_{i}$ for $i \in E^{\prime}$. 
Example 1. A MAP with phase-type jumps can be transformed into an MMBM, as shown in detail in [6, Section 2.1]. The resulting MMBM has a phase space $E=E_{+} \cup E_{p} \cup E_{\sigma} \cup E_{n} \cup E_{-}$, where

$$
\begin{aligned}
& E_{p}=\left\{i \in \tilde{E}: \tilde{\mu}_{i}>0, \tilde{\sigma}_{i}=0\right\}, \\
& E_{n}=\left\{i \in \tilde{E}: \tilde{\mu}_{i}<0, \tilde{\sigma}_{i}=0\right\}, \\
& E_{\sigma}=\left\{i \in \tilde{E}: \tilde{\sigma}_{i}>0\right\},
\end{aligned}
$$

and phases in $E_{ \pm}$represent parts of the jumps (see [6, Section 2.1] for a precise definition). In order to retrieve the Laplace transform of the first passage times of the original MAP (i.e. the one with phase-type jumps), it suffices to set $r_{i}:=0$ for $i \in E_{+} \cup E_{-}$and $r_{i}:=\gamma$ for $i \in E_{p} \cup E_{\sigma} \cup E_{n}$. This method is called fluid embedding and has been described in Section 2.2 of [6], Section 2.7 of [11], and Section 3 of [13].

Example 2. We shall derive the joint Laplace transform of the ruin time and the accumulated claims for the classical compound Poisson risk model. Denote the initial risk reserve by $u \geq 0$. The claim sizes and interclaim times will be independent and have exponential distributions with parameters $\beta>0$ and $\lambda>0$, respectively. The rate of premium income is denoted by $c>0$. This model has been analysed in [8]. The net profit condition is $c / \lambda>1 / \beta$, which is equivalent to $\lambda /(c \beta)<1$.

We consider an MMBM $(\mathcal{X}, \mathcal{g})$ which is defined as follows. Let the phase space be given by $E=\{1,2\}$. The parameters are given by $\sigma_{1}=\sigma_{2}=0, \mu_{1}=1, \mu_{2}=-c$, and

$$
Q=\left(\begin{array}{cc}
-\beta & \beta \\
\lambda & -\lambda
\end{array}\right)
$$

Then the ruin time $T(u)$ for the compound Poisson model coincides with the occupation time in phase 2 until the first passage time $\tau(u)$, given that we start with $X_{0}=0$. Likewise, the accumulated claim until ruin, denoted by $D(u)$, coincides with the occupation time in phase 1 until $\tau(u)$. The joint Laplace transform of $D(u)$ and $T(u)$ with arguments $r_{1}$ and $r_{2}$, respectively, is given by

$$
\mathrm{E}\left(\mathrm{e}^{-r_{1} D(u)} \mathrm{e}^{-r_{2} T(u)} \mid X_{0}=0, J_{0}=2\right)=e_{2}^{\prime} \mathrm{E}\left(\exp \left[-\int_{0}^{\tau(u)} r_{J_{s}} \mathrm{~d} s\right]\right) \mathbf{1}=A(\boldsymbol{r}) \mathrm{e}^{U(\boldsymbol{r}) u},
$$

where $A(\boldsymbol{r})$ and $U(\boldsymbol{r})$ are real numbers. They can be computed using (3) and (5) as the fixed points

$$
U(\boldsymbol{r})=-\left(\beta+r_{1}\right)+\beta A(\boldsymbol{r}) \quad \text { and } \quad A(\boldsymbol{r})=\lambda\left(\lambda+r_{2}-c U(\boldsymbol{r})\right)^{-1}
$$

with minimal positive solution

$$
A(\boldsymbol{r})=\frac{1}{2 c \beta}\left(\lambda+r_{2}+c\left(\beta+r_{1}\right)-\sqrt{\left(\lambda+r_{2}+c\left(\beta+r_{1}\right)\right)^{2}-4 \lambda c \beta}\right),
$$

from which $U(\boldsymbol{r})$ can be readily computed. For $r_{1}=0$, we obtain the Laplace transform of the time of ruin, for which the result is the same as Equation (5.38) of [8]; cf. Example 5 of [5].

\subsection{Occupation times before an exit from an interval}

For $l<u$, define $\tau(l, u):=\inf \left\{t \geq 0: X_{t} \notin[l, u]\right\}$, which is the exit time of $\mathcal{X}$ from the interval $[l, u]$. We shall need an expression for

$$
\Psi_{i j}^{+}(l, u \mid x):=\mathrm{E}\left(\exp \left[-\int_{0}^{\tau(l, u)} r_{J_{s}} \mathrm{~d} s\right] ; X_{\tau(l, u)}=u, J_{\tau(l, u)}=j \mid J_{0}=i, X_{0}=x\right),
$$


where $x \in[l, u]$ and $i, j \in E$. Define the matrix $\Psi^{+}(l, u \mid x):=\left(\Psi_{i j}^{+}(l, u \mid x)\right)_{i, j \in E \text {. }}$ A formula for $\Psi^{+}(l, u \mid x)$ has been derived in [13]. In order to state it, we need some additional notation. In order to simplify this notation, we shall from now on exclude the case of a phase $i \in E$ with $\mu_{i}=\sigma_{i}=0$.

Let $\left(\mathcal{X}^{+}, \mathcal{g}\right)$ denote the original MMBM, and define the process $\left(\mathcal{X}^{-}, g\right) \stackrel{\mathrm{D}}{=}\left(-\mathcal{X}^{+}, \boldsymbol{g}\right)$, where $\stackrel{\text { D }}{=}$ denotes equality in distribution. The two processes have the same generator matrix $Q$ for $g$, but the drift parameters are different. Denoting the variation and drift parameters for $\mathcal{X}^{ \pm}$by $\sigma_{i}^{ \pm}$and $\mu_{i}^{ \pm}$, respectively, this means that $\sigma_{i}^{-}=\sigma_{i}^{+}$and $\mu_{i}^{-}=-\mu_{i}^{+}$for all $i \in E$.

Let $A^{ \pm}(\boldsymbol{r})$ and $U^{ \pm}(\boldsymbol{r})$ denote the matrices that determine the first passage times of $X^{ \pm}$ in (2). We write $A^{ \pm}=A^{ \pm}(\boldsymbol{r})$ and $U^{ \pm}=U^{ \pm}(\boldsymbol{r})$ when we do not wish to emphasize the dependence on $\boldsymbol{r}$. We split the ascending phases into the spaces $E_{s}:=\left\{i \in E_{a}: \sigma_{i}=0\right\}$ and $E_{\sigma}:=\left\{i \in E_{a}: \sigma_{i}>0\right\}$, and let $I_{s}$ and $I_{\sigma}$ respectively denote the identity matrices on $E_{s}$ and $E_{\sigma}$. We call a phase $i \in E_{s}$ strictly ascending. Define the matrices

$$
C^{+}:=C^{+}(\boldsymbol{r}):=\left(\begin{array}{ll}
\mathbf{0} & I_{\sigma} \\
A^{+} & (\boldsymbol{r})
\end{array}\right) \quad \text { and } \quad C^{-}:=C^{-}(\boldsymbol{r}):=\left(\begin{array}{cr}
A^{-}(\boldsymbol{r}) \\
I_{\sigma} & \mathbf{0}
\end{array}\right)
$$

of dimensions $\left(E_{\sigma} \cup E_{d}\right) \times E_{a}$ and $E_{a} \times\left(E_{\sigma} \cup E_{d}\right)$, respectively. Furthermore, define

$$
W^{+}:=W^{+}(\boldsymbol{r}):=\left(\begin{array}{c}
I_{a} \\
A^{+}(\boldsymbol{r})
\end{array}\right) \quad \text { and } \quad W^{-}:=W^{-}(\boldsymbol{r}):=\left(\begin{array}{cc}
A^{-}(\boldsymbol{r}) \\
I_{\sigma} & \mathbf{0} \\
\mathbf{0} & I_{d}
\end{array}\right) \text {, }
$$

which are matrices of dimensions $E \times E_{a}$ and $E \times\left(E_{\sigma} \cup E_{d}\right)$. Finally, let

$$
Z^{ \pm}:=C^{ \pm} \mathrm{e}^{U^{ \pm} \cdot(u-l)}
$$

Then Equation (23) of [13] states that

$$
\Psi^{+}(l, u \mid x)=\left(W^{+} \mathrm{e}^{U^{+} \cdot(u-x)}-W^{-} \mathrm{e}^{U^{-} \cdot(x-l)} C^{+} \mathrm{e}^{U^{+} \cdot(u-l)}\right) \cdot\left(I-Z^{-} Z^{+}\right)^{-1}
$$

for $l \leq x \leq u$. By reflection at the initial level $x$, we obtain, from (7),

$$
\begin{aligned}
\Psi^{-}(l, u \mid x) & :=\mathrm{E}\left(\exp \left[-\int_{0}^{\tau(l, u)} r_{J_{s}} \mathrm{~d} s\right] ; X_{\sigma(l, u)}=l \mid X_{0}=x\right) \\
& =\left(W^{-} \mathrm{e}^{U^{-} \cdot(x-l)}-W^{+} \mathrm{e}^{U^{+} \cdot(u-x)} C^{-} \mathrm{e}^{U^{-} \cdot(u-l)}\right) \cdot\left(I-Z^{+} Z^{-}\right)^{-1}
\end{aligned}
$$

for $l \leq x \leq u$. Note that the expressions in (7) and (8) depend on a choice of $\boldsymbol{r}$.

Example 3. (Example 2 continued.) We obtain $A^{-}(\boldsymbol{r})$ and $U^{-}(\boldsymbol{r})$ by solving

$$
U^{-}(\boldsymbol{r})=-\frac{\lambda+r_{2}}{c}+\frac{\lambda}{c} A^{-}(\boldsymbol{r}) \quad \text { and } \quad A^{-}(\boldsymbol{r})=\beta\left(\beta+r_{1}-U^{-}(\boldsymbol{r})\right)^{-1} .
$$

This yields

$$
A^{-}(\boldsymbol{r})=\frac{1}{2 \lambda}\left(\left(c\left(\beta+r_{1}\right)+\lambda+r_{2}\right)-\sqrt{\left(c\left(\beta+r_{1}\right)+\lambda+r_{2}\right)^{2}-4 c \beta}\right),
$$

whence $U^{-}(\boldsymbol{r})$ may be readily obtained. 


\section{Some remarks on scale functions}

Noting that $\left(I-Z^{-} Z^{+}\right)^{-1}=\sum_{n=0}^{\infty}\left(Z^{-} Z^{+}\right)^{n}$ and $Z^{-} Z^{+}$represents a crossing over the interval $[l, u]$ from $u$ to $l$ and back, (7) has a clear probabilistic interpretation. The term $W^{+} \mathrm{e}^{U^{+} \cdot(u-x)}$ simply yields the event that $\mathcal{X}$ exits from $u$. The correction term $W^{-} \mathrm{e}^{U^{-} \cdot x} Z^{+}$ refers to the event that $\mathcal{X}$ descends below $l$ before exiting from $u$. Multiplication by ( $I-$ $\left.Z^{-} Z^{+}\right)^{-1}$ yields all possible combinations with any number of subsequent (down and up) crossings over the complete interval $[l, u]$.

Since $Z^{+}=C^{+} \mathrm{e}^{U^{+} \cdot(u-l)}$, we can write $\Psi^{+}(l, u \mid x)$ in the form

$$
\Psi^{+}(l, u \mid x)=\left(W^{+} \mathrm{e}^{-U^{+} \cdot(x-l)}-W^{-} \mathrm{e}^{U^{-} \cdot(x-l)} C^{+}\right)\left(\mathrm{e}^{-U^{+} \cdot(u-l)}-C^{-} \mathrm{e}^{U^{-} \cdot(u-l)} C^{+}\right)^{-1} .
$$

This comes closer to the usual expression of the exit time distribution in terms of scale functions. For instance, let $\mathcal{X}$ be a Brownian motion with variation $\sigma>0$ and drift $\mu \in \mathbb{R}$. We then obtain

$$
U^{ \pm}=\frac{ \pm \mu-\sqrt{\mu^{2}+2 \gamma \sigma^{2}}}{\sigma^{2}} .
$$

Define $r:=-U^{+}$and $s:=U^{-}$. Then

$$
\Psi^{+}(0, b \mid x)=\frac{\mathrm{e}^{r x}-\mathrm{e}^{s x}}{\mathrm{e}^{r b}-\mathrm{e}^{s b}}
$$

cf. [9, Equations (2.12)-(2.15)], where the $\gamma$-scale function is given as $g(x)=\mathrm{e}^{r x}-\mathrm{e}^{s x}$.

As we can see from (9), scale functions as solutions to the two-sided exit problem are determined only up to a multiplicative constant. The usual unique definition of the $\gamma$-scale function $W^{(\gamma)}(x)$ for a Lévy process with cumulant function $\psi$ is in terms of its Laplace transform

$$
\int_{0}^{\infty} \mathrm{e}^{-\beta x} W^{(\gamma)}(x) \mathrm{d} x=\frac{1}{\psi(\beta)-\gamma}
$$

for $\beta>\Phi(\gamma)$, where $\Phi$ denotes the right inverse of $\psi$; see Equation (8.5) of [14].

For the case of an MMBM with $\sigma_{i}>0$ for all $i \in E$, we can extend the notion of $\gamma$-scale functions. In this case there are no matrices $A^{ \pm}$and, thus, $W^{ \pm}=C^{ \pm}=I$. For a vector $v=\left(v_{1}, \ldots, v_{m}\right)$, define the diagonal matrix with entries taken from $v$ by $\Delta_{v}:=\operatorname{diag}\left(v_{i}\right)_{i \in E}$. With $\sigma^{2}:=\left(\sigma_{1}^{2}, \ldots, \sigma_{m}^{2}\right)$ and $\mu:=\left(\mu_{1}, \ldots, \mu_{m}\right)$, we obtain, by the same arguments as for Equation (5) of [5],

$$
\Delta_{\boldsymbol{r}}=\Delta_{\sigma^{2} / 2} U(\boldsymbol{r})^{2}-\Delta_{\mu} U(\boldsymbol{r})+Q
$$

(use the function in (1) instead of $f_{i j}(x)$ defined in Equation (3) of [5]). Note that there is a typo in Equation (6) of [5]: $+\Delta_{\mu}$ should read $-\Delta_{\mu}$. Define the scalar cumulant functions $\psi_{i}(\beta):=\sigma_{i}^{2} / 2 \beta^{2}+\mu_{i} \beta$ for $i \in E$, and write $\psi(\beta):=\left(\psi_{1}(\beta), \ldots, \psi_{m}(\beta)\right)$. Then the (matrixvalued) cumulant function of $\left(\mathcal{X}^{+}, \mathcal{g}\right)$ is given as $K(\beta)=\Delta_{\psi(\beta)}+Q$; see Proposition XI.2.2 of [2]. This yields

$$
\begin{aligned}
K(\beta)-\Delta_{\boldsymbol{r}} & =\Delta_{\sigma^{2} / 2}\left(\beta^{2} I-U_{+}^{2}\right)+\Delta_{\mu}\left(\beta I+U_{+}\right) \\
& =\left(\Delta_{\sigma^{2} / 2}\left(\beta I-U_{+}\right)+\Delta_{\mu}\right)\left(\beta I+U_{+}\right),
\end{aligned}
$$

where we have set $U_{+}=U^{+}(\boldsymbol{r})$. Similarly, for the negative process $\left(\mathcal{X}^{-}, \boldsymbol{g}\right)$, we obtain

$$
\Delta_{\boldsymbol{r}}=\Delta_{\sigma^{2} / 2} U^{-}(\boldsymbol{r})^{2}+\Delta_{\mu} U^{-}(\boldsymbol{r})+Q,
$$


and, hence,

$$
\begin{aligned}
K(\beta)-\Delta_{\boldsymbol{r}} & =\Delta_{\sigma^{2} / 2}\left(\beta^{2} I-U_{-}^{2}\right)+\Delta_{\mu}\left(\beta I-U_{-}\right) \\
& =\left(\Delta_{\sigma^{2} / 2}\left(\beta I+U_{-}\right)+\Delta_{\mu}\right)\left(\beta I-U_{-}\right),
\end{aligned}
$$

where we have set $U_{-}=U^{-}(\boldsymbol{r})$. We propose calling

$$
W^{(\boldsymbol{r})}(x)=\left(\mathrm{e}^{-U_{+} \cdot x}-\mathrm{e}^{U_{-} \cdot x}\right) \cdot C, \quad x \geq 0,
$$

the $\boldsymbol{r}$-scale function of $(\mathcal{X}, \boldsymbol{g})$, where the constant $C$ remains to be determined. Let $\left\|U_{+}\right\|$ denote the largest absolute value of any eigenvalue of $U_{+}$. For $\beta>\left\|U_{+}\right\|$, we evaluate

$$
\begin{aligned}
(K(\beta) & \left.-\Delta_{\boldsymbol{r}}\right) \int_{0}^{\infty} \mathrm{e}^{-\beta x} W^{(\boldsymbol{r})}(x) \mathrm{d} x \\
& =\left(K(\beta)-\Delta_{\boldsymbol{r}}\right)\left(\left(\beta I+U_{+}\right)^{-1}-\left(\beta I-U_{-}\right)^{-1}\right) C \\
& =\left(\Delta_{\sigma^{2} / 2}\left(\beta I-U_{+}\right)+\Delta_{\mu}-\Delta_{\sigma^{2} / 2}\left(\beta I+U_{-}\right)-\Delta_{\mu}\right) C, \\
& =-\Delta_{\sigma^{2} / 2}\left(U_{+}+U_{-}\right) C,
\end{aligned}
$$

where the second equality follows from (10) and (11). With $C:=-\left(U_{+}+U_{-}\right)^{-1} \cdot \Delta_{2 / \sigma^{2}}$ we thus obtain

$$
\left(K(\beta)-\Delta_{\boldsymbol{r}}\right) \int_{0}^{\infty} \mathrm{e}^{-\beta x} W^{(\boldsymbol{r})}(x) \mathrm{d} x=I
$$

for $\beta>\left\|U_{+}\right\|$, which justifies the name ' $r$-scale function'.

Remark 2. In order to compare the above proposal with results obtained in [11, Section 7.5], we first translate the notation $U_{+}=\Lambda$ and $\boldsymbol{r}=q \cdot \mathbf{1}$. Furthermore, note that $\mathrm{e}^{U_{-} \cdot x}=\mathrm{P}\left(J\left(\tau^{\{-x\}}\right)\right)$ and $\Pi=I$, since $E=E_{\sigma}$. Thus, Equation (7.7) of [11] translates as $\tilde{W}(x)=\mathrm{e}^{-U_{+} \cdot x}-\mathrm{e}^{U_{-} \cdot x}$. Moreover, Equation (7.9) of [11] together with the above determination of the matrix $C$ yields the expression $\boldsymbol{L}=-\left(U_{+}+U_{-}\right)^{-1} \cdot \Delta_{2 / \sigma^{2}}$ for the matrix of expected local times at 0 . Equation (7.4) of [11] then leads to the expression

$$
\boldsymbol{L}^{q}(x)=-\left(I-\mathrm{e}^{U_{+} \cdot x} \mathrm{e}^{U_{-} \cdot x}\right) \cdot\left(U_{+}+U_{-}\right)^{-1} \cdot \Delta_{2 / \sigma^{2}}
$$

for the matrix of expected local times at 0 before the first passage over a level $x \geq 0$.

\section{Occupation times for level and phase processes in two intervals}

While the occupation times for the phase process have been obtained in [6] and [13], with some translations given in Section 2, the more interesting (and more difficult) part of our investigation is determining the occupation times of the level process in different intervals. Their distribution will be derived in this section for the case of two contiguous intervals. A general recursion scheme for more than two intervals will be provided in Section 5.

\subsection{Occupation times before an exit from an interval}

Recall the definition of the exit times of $\mathcal{X}$ from an interval $[l, u]$, namely,

$$
\tau(l, u):=\inf \left\{t \geq 0: X_{t}<l \text { or } X_{t}>u\right\},
$$

where $X_{0} \in[u, l]$. Choose some $b \in(l, u)$, and define, for $j \in E$,

$$
\zeta_{1, j}(l, u):=\int_{0}^{\tau(l, u)} \mathbf{1}_{\left\{X_{t}<b, J_{t}=j\right\}} \mathrm{d} t \quad \text { and } \quad \zeta_{2, j}(l, u):=\int_{0}^{\tau(l, u)} \mathbf{1}_{\left\{X_{t}>b, J_{t}=j\right\}} \mathrm{d} t .
$$


Furthermore, define the column vectors $\zeta_{k}(l, u):=\left(\zeta_{k j}(l, u): j \in E\right)$ for $k \in\{1,2\}$. The random variables $\zeta_{1 j}(l, u)$ and $\zeta_{2 j}(l, u)$ yield the occupation times of $(\mathcal{X}, \mathcal{g})$ in the sets $[l, b) \times$ $\{j\}$ and $(b, u] \times\{j\}, j \in E$, before the level process leaves the interval $[l, u]$.

Choose any exit rate vectors $\boldsymbol{r}_{k}=\left(r_{k j}: j \in E\right)$ for $k \in\{1,2\}$. We shall derive an expression for

$$
E^{+}(l, u \mid a):=\mathrm{E}\left(\mathrm{e}^{-r_{1} \zeta_{1}(l, u)} \mathrm{e}^{-r_{2} \zeta_{2}(l, u)} ; X_{\tau(l, u)}=u \mid X_{0}=a\right),
$$

where $l<a<u$. This provides the joint Laplace transform of the occupation times $\zeta_{k j}(l, u)$ before the first exit of $[l, u]$, restricted to the exit occurring at $u$.

There are some simple cases. For $l<a<b<u$, we obtain

$$
E^{+}(l, u \mid a)=\Psi_{r_{1}}^{+}(l, b \mid a) E^{+}(l, u \mid b)
$$

by path continuity, and, similarly, for $l<b<a<u$, we observe that

$$
E^{+}(l, u \mid a)=\Psi_{r_{2}}^{+}(b, u \mid a)+\Psi_{r_{2}}^{-}(b, u \mid a) E^{+}(l, u \mid b) .
$$

Thus, it suffices to determine $E^{+}(l, u \mid b)$. For any matrix $M$ of dimension $E \times E$, write

$$
M=:\left(\begin{array}{ll}
M_{(a, a)} & M_{(a, d)} \\
M_{(d, a)} & M_{(d, d)}
\end{array}\right)=:\left(M_{(\cdot, a)}, M_{(\cdot, d)}\right),
$$

thereby distinguishing between the ascending $\left(E_{a}\right)$ and descending $\left(E_{d}\right)$ phases. Clearly, $E_{(\cdot, d)}^{+}(l, u \mid b)=\mathbf{0}$, since $u$ cannot be passed from below in a descending phase. Discerning between initial phases, we find that

$$
E_{(d, a)}^{+}(l, u \mid b)=\Psi_{r_{1}}^{+}(l, b \mid b)_{(d, a)} E_{(a, a)}^{+}(l, u \mid b)
$$

such that it remains to determine $E_{(a, a)}^{+}(l, u \mid b)$. For a matrix $M$ of dimension $E_{a} \times E_{a}$, write

$$
M=:\left(\begin{array}{ll}
M_{(s, s)} & M_{(s, \sigma)} \\
M_{(\sigma, s)} & M_{(\sigma, \sigma)}
\end{array}\right)=:\left(\begin{array}{l}
M_{(s, \cdot)} \\
M_{(\sigma, \cdot)}
\end{array}\right)
$$

in obvious block notation. Conditioning on the number $n$ of possible returns to the level $b$ in a strictly ascending phase (i.e. one from $E_{s}$ ) before exiting the interval $[l, u]$ at $u$, we observe that

$$
\begin{aligned}
E_{(s, \cdot)}^{+}(l, u \mid b) & =\sum_{n=0}^{\infty}\left(\Psi_{\boldsymbol{r}_{2}}^{-} \Psi_{\boldsymbol{r}_{1}}^{+}\right)_{(s, s)}^{n}\left(\left(\Psi_{\boldsymbol{r}_{2}}^{+}\right)_{(s, \cdot)}+\left(\Psi_{\boldsymbol{r}_{2}}^{-} \Psi_{\boldsymbol{r}_{1}}^{+}\right)_{(s, \sigma)} E_{(\sigma, \cdot)}^{+}(l, u \mid b)\right) \\
& =\left(I_{s}-\left(\Psi_{\boldsymbol{r}_{2}}^{-} \Psi_{\boldsymbol{r}_{1}}^{+}\right)_{(s, s)}\right)^{-1}\left(\left(\Psi_{\boldsymbol{r}_{2}}^{+}\right)_{(s, \cdot)}+\left(\Psi_{\boldsymbol{r}_{2}}^{-} \Psi_{\boldsymbol{r}_{1}}^{+}\right)_{(s, \sigma)} E_{(\sigma, \cdot)}^{+}(l, u \mid b)\right),
\end{aligned}
$$

where $I_{s}$ denotes the identity matrix on $E_{s}, \Psi_{\boldsymbol{r}_{2}}^{ \pm}=\Psi_{\boldsymbol{r}_{2}}^{ \pm}(b, u \mid b)$, and $\Psi_{\boldsymbol{r}_{1}}^{+}$denotes the $\left(E_{\sigma} \cup\right.$ $\left.E_{d}\right) \times E_{a}$ block of $\Psi_{r_{1}}^{+}(l, b \mid b)$. We have thus reduced the problem to the determination of $E_{(\sigma, \cdot)}^{+}(l, u \mid b)$.

Theorem 1. Write $U_{k}^{ \pm}:=U^{ \pm}\left(\boldsymbol{r}_{k}\right)$ for $k \in\{1,2\}$. For $l<b<u$,

$$
\begin{aligned}
E_{(\sigma, a)}^{+}(l, u \mid b)= & 2\left(\left(D_{1}\right)_{(\sigma, \sigma)}+\left(D_{2} \Psi_{r_{1}}^{+}(l, b \mid b)\right)_{(\sigma, \sigma)}\right)^{-1} \\
& \times\left(\mathbf{0}_{(\sigma, s)}, I_{\sigma}\right)\left(U_{2}^{+}+C_{2}^{-} U_{2}^{-} C_{2}^{+}\right)\left(\mathrm{e}^{-U_{2}^{+} \cdot(u-b)}-C_{2}^{-} \mathrm{e}^{U_{2}^{-} \cdot(u-b)} C_{2}^{+}\right)^{-1},
\end{aligned}
$$

where the constant matrices $D_{1}$ and $D_{2}$ are given in Lemmata 1 and 2. 
Proof. We employ the following approximation. Assume that the exit rate vector changes from $\boldsymbol{r}_{2}$ to $\boldsymbol{r}_{1}$ at $b-\varepsilon$ for downward crossings of $b$ and from $\boldsymbol{r}_{1}$ to $\boldsymbol{r}_{2}$ at $b+\varepsilon$ for upward crossings. Then we let $\varepsilon \downarrow 0$.

To be more precise, assume that $X_{0}=b+\varepsilon$, and define $t_{0}:=0$ as well as the times $s_{n}:=\min \left\{t>t_{n-1}: X_{t}=b-\varepsilon\right\}$ and $t_{n}:=\min \left\{t>s_{n}: X_{t}=b+\varepsilon\right\}$ for all $n \in \mathbb{N}$, where $\min \varnothing:=\infty$. Let $N:=\max \left\{n \in \mathbb{N}_{0}: t_{n}<\tau(l, u)\right\}$. Note that, on $\left\{X_{\tau(l, u)}=u\right\}$, for each $s_{n}<\tau(l, u)$, there exists a $t_{n}$ such that $s_{n}<t_{n}<\tau(l, u)$ due to path continuity. We consider

$$
\begin{gathered}
E(\varepsilon):=\mathrm{E}_{(\sigma, a)}\left(\exp \left[-\sum_{n=1}^{N} \int_{t_{n-1}}^{s_{n}} \boldsymbol{r}_{2} e_{J_{s}} \mathrm{~d} s-\int_{t_{N}}^{\tau(l, u)} \boldsymbol{r}_{2} e_{J_{s}} \mathrm{~d} s\right] \exp \left[-\sum_{n=1}^{N} \int_{s_{n}}^{t_{n}} \boldsymbol{r}_{1} e_{J_{s}} \mathrm{~d} s\right] ;\right. \\
\left.X_{\tau(l, u)}=u \mid X_{0}=b+\varepsilon\right) .
\end{gathered}
$$

This converges to

$$
\begin{aligned}
\mathrm{E}_{(\sigma, a)}\left(\exp \left[-\int_{0}^{\tau(l, u)} \boldsymbol{r}_{1} e_{J_{s}} \mathbf{1}_{\left\{X_{s}<b\right\}} \mathrm{d} s\right] \exp \left[-\int_{0}^{\tau(l, u)} \boldsymbol{r}_{2} e_{J_{s}} \mathbf{1}_{\left\{X_{s}>b\right\}} \mathrm{d} s\right]\right. \\
\left.X_{\tau(l, u)}=u \mid X_{0}=b\right) \\
=E_{(\sigma, a)}^{+}(l, u \mid b)
\end{aligned}
$$

as $\varepsilon \downarrow 0$, since

$$
\lim _{\varepsilon \downarrow 0} \int_{0}^{\tau(l, u)} \mathbf{1}_{\left\{b-\varepsilon<X_{t}<b+\varepsilon\right\}} \mathrm{d} t=0
$$

almost surely.

Write $\Psi_{2}^{-}(\varepsilon)$ for the $\left(E_{a} \times\left(E_{\sigma} \cup E_{d}\right)\right)$-block of $\Psi_{\boldsymbol{r}_{2}}^{-}(b-\varepsilon, u \mid b+\varepsilon)$ and $\Psi_{2}^{+}(\varepsilon)$ for the $\left(E_{a} \times E_{a}\right)$-block of $\Psi_{\boldsymbol{r}_{2}}^{+}(b-\varepsilon, u \mid b+\varepsilon)$. Furthermore, write $\Psi_{1}^{+}(\varepsilon)$ for the $\left(\left(E_{\sigma} \cup E_{d}\right) \times E_{a}\right)$ block of $\Psi_{r_{1}}^{+}(l, b+\varepsilon \mid b-\varepsilon)$. Summing over the number of down and up crossings of the interval $[b-\varepsilon, b+\varepsilon]$ before leaving the interval $[l, u]$ at $u$, we obtain

$$
\begin{aligned}
E(\varepsilon) & =\left(\mathbf{0}_{(\sigma, s)}, I_{\sigma}\right) \sum_{n=0}^{\infty}\left(\Psi_{2}^{-}(\varepsilon) \Psi_{1}^{+}(\varepsilon)\right)^{n} \Psi_{2}^{+}(\varepsilon) \\
& =\left(\mathbf{0}_{(\sigma, s)}, I_{\sigma}\right)\left(I_{a}-\Psi_{2}^{-}(\varepsilon) \Psi_{1}^{+}(\varepsilon)\right)^{-1} I(\varepsilon) I(\varepsilon)^{-1} \Psi_{2}^{+}(\varepsilon),
\end{aligned}
$$

where

$$
I(\varepsilon):=\left(\begin{array}{cc}
I_{s} & \mathbf{0} \\
\mathbf{0} & \varepsilon I_{\sigma}
\end{array}\right) .
$$

First we consider $\lim _{\varepsilon \downarrow 0}\left(\mathbf{0}_{(\sigma, s)}, I_{\sigma}\right)\left(I_{a}-\Psi_{2}^{-}(\varepsilon) \Psi_{1}^{+}(\varepsilon)\right)^{-1} I(\varepsilon)$. Since

$$
\lim _{\varepsilon \downarrow 0}\left(\mathbf{0}_{(\sigma, s)}, I_{\sigma}\right) \Psi_{2}^{-}(\varepsilon) \Psi_{1}^{+}(\varepsilon)=\left(I_{\sigma}, \mathbf{0}_{(\sigma, d)}\right) \Psi_{1}^{+}(\varepsilon)=\left(\mathbf{0}_{(\sigma, s)}, I_{\sigma}\right),
$$

we find that $\lim _{\varepsilon \downarrow 0} \Psi_{2}^{-}(\varepsilon) \Psi_{1}^{+}(\varepsilon)$ is an upper triagonal block matrix. We thus obtain

$$
\lim _{\varepsilon \downarrow 0}\left(\mathbf{0}_{(\sigma, s)}, I_{\sigma}\right)\left(I_{a}-\Psi_{2}^{-}(\varepsilon) \Psi_{1}^{+}(\varepsilon)\right)^{-1} I(\varepsilon)=\left(\mathbf{0}_{(\sigma, s)}, D\right)
$$


for an $E_{\sigma} \times E_{\sigma}$ matrix $D$ that is given by

$$
\begin{aligned}
D & =\lim _{\varepsilon \downarrow 0} \varepsilon\left(I_{\sigma}-\left(\Psi_{2}^{-}(\varepsilon) \Psi_{1}^{+}(\varepsilon)\right)_{(\sigma, \sigma)}\right)^{-1} \\
& =-\left(\lim _{\varepsilon \downarrow 0} \frac{1}{\varepsilon}\left(\left(\Psi_{2}^{-}(\varepsilon) \Psi_{1}^{+}(\varepsilon)\right)_{(\sigma, \sigma)}-I_{\sigma}\right)\right)^{-1} \\
& =-\left(\left.\frac{\mathrm{d}}{\mathrm{d} \varepsilon}\left(\Psi_{2}^{-}(\varepsilon) \Psi_{1}^{+}(\varepsilon)\right)_{(\sigma, \sigma)}\right|_{\varepsilon=0}\right)^{-1} \\
& =-\left(\left(\left.\frac{\mathrm{d}}{\mathrm{d} \varepsilon} \Psi_{2}^{-}(\varepsilon)\right|_{\varepsilon=0} \Psi_{1}^{+}(0)\right)_{(\sigma, \sigma)}+\left(\left.\Psi_{2}^{-}(0) \frac{\mathrm{d}}{\mathrm{d} \varepsilon} \Psi_{1}^{+}(\varepsilon)\right|_{\varepsilon=0}\right)_{(\sigma, \sigma)}\right)^{-1} \\
& =-\left(\left(D_{2} \Psi_{1}^{+}(0)\right)_{(\sigma, \sigma)}+\left(D_{1}\right)_{(\sigma, \sigma)}\right)^{-1} ;
\end{aligned}
$$

see [4, Section I.1.3] as well as Lemmata 1 and 2 for the last two equalities. In a similar manner, since $\lim _{\varepsilon \downarrow 0} \Psi_{2}^{+}(\varepsilon)_{(\sigma, a)}=\mathbf{0}$, we obtain

$$
\lim _{\varepsilon \downarrow 0} \varepsilon^{-1} \Psi_{2}^{+}(\varepsilon)_{(\sigma, a)}=\left.\frac{\mathrm{d}}{\mathrm{d} \varepsilon} \Psi_{2}^{+}(\varepsilon)_{(\sigma, a)}\right|_{\varepsilon=0}=D_{3}
$$

according to Lemma 3. Altogether, this yields the expression in the statement.

Example 4. Consider a finite buffer with capacity $u>0$. The buffer content is modelled by a Brownian motion with parameters $\mu<0$ (drift) and $\sigma>0$ (variation). This corresponds to a phase space $E=E_{\sigma}=\{1\}$ consisting of one element only. Thus, $C^{ \pm}=1$.

Assume that there is a level $b \in(0, u)$ above which there is a higher cost attached. We wish to compute the Laplace transform of the time spent above the level $b$ along with the probability of a buffer overflow. To shorten considerations, we assume that the initial buffer content is $b$. Then the Laplace transform we aim for can be computed as $E^{+}(0, b, u)$ with exit rate vectors $\boldsymbol{r}_{1}=0$ and $\boldsymbol{r}_{2}=\gamma$, where $\gamma$ is the argument for the Laplace transform. Thus, we need to determine only $U_{1}^{ \pm}$and $U_{2}^{ \pm}$, which are real numbers. We obtain

$$
U_{k}^{ \pm}=-\frac{1}{\sigma} \sqrt{2 \boldsymbol{r}_{k}+\frac{\mu^{2}}{\sigma^{2}}} \pm \frac{\mu}{\sigma^{2}}
$$

for $k \in\{1,2\}$, according to (4). Since $\mu<0$, this yields $U_{1}^{+}=2 \mu / \sigma^{2}$ and $U_{1}^{-}=0$. Hence,

$$
D_{1}=2 \frac{2 \mu / \sigma^{2}}{1-\mathrm{e}^{2 \mu / \sigma^{2} \cdot b}},
$$

and, upon setting $W:=(2 / \sigma) \sqrt{2 \gamma+\mu^{2} / \sigma^{2}}$,

$$
\begin{aligned}
E^{+}(0, u \mid b)= & \left(\frac{2 \mu / \sigma^{2}}{1-\mathrm{e}^{2 \mu / \sigma^{2} \cdot b}}+\frac{U_{2}^{-} \mathrm{e}^{-U_{2}^{-} \cdot(u-b)}+U_{2}^{+} \mathrm{e}_{2}^{U_{2}^{+} \cdot(u-b)}}{\mathrm{e}^{-U_{2}^{-} \cdot(u-b)}-\mathrm{e}_{2}^{U^{+} \cdot(u-b)}}\right)^{-1} \\
& \times \frac{U_{2}^{+}+U_{2}^{-}}{\mathrm{e}^{-U_{2}^{+} \cdot(u-b)}-\mathrm{e}_{2}^{U_{2}^{-} \cdot(u-b)}} \\
= & \left(\frac{-2 \mu / \sigma^{2}}{1-\mathrm{e}^{2 \mu / \sigma^{2} \cdot b}}+\frac{-U_{2}^{-}-U_{2}^{+} \mathrm{e}^{-W \cdot(u-b)}}{1-\mathrm{e}^{-W \cdot(u-b)}}\right)^{-1} \frac{W}{1-\mathrm{e}^{-W \cdot(u-b)}} \mathrm{e}^{U_{2}^{+} \cdot(u-b)} \\
= & \left(\frac{-2 \mu / \sigma^{2}}{1-\mathrm{e}^{2 \mu / \sigma^{2} \cdot b}}+\frac{W}{1-\mathrm{e}^{-W \cdot(u-b)}}+U_{2}^{+}\right)^{-1} \frac{W}{1-\mathrm{e}^{-W \cdot(u-b)}} \mathrm{e}^{U_{2}^{+} \cdot(u-b)}
\end{aligned}
$$

since $U_{2}^{+}+U_{2}^{-}=-(2 / \sigma) \sqrt{2 \gamma+\mu^{2} / \sigma^{2}}=-W$. 
Example 5. Considering Brownian motion as in the previous example, but this time with exit rates $\boldsymbol{r}_{1}=\boldsymbol{r}_{2}=\gamma$, we obtain $U_{1}^{ \pm}=U_{2}^{ \pm}=: u^{ \pm}$. This yields

$$
D_{1}=2 \frac{u^{+} \mathrm{e}^{-u^{+} \cdot(b-l)}+u^{-} \mathrm{e}^{u^{-} \cdot(b-l)}}{\mathrm{e}^{-u^{+} \cdot(b-l)}-\mathrm{e}^{u^{-} \cdot(b-l)}}
$$

and

$$
D_{2}=2 \frac{u^{-} \mathrm{e}^{-u^{-} \cdot(u-b)}+u^{+} \mathrm{e}^{u^{+} \cdot(u-b)}}{\mathrm{e}^{-u^{-} \cdot(u-b)}-\mathrm{e}^{u^{+} \cdot(u-b)}}=2 \frac{u^{-} \mathrm{e}^{-u^{+} \cdot(u-b)}+u^{+} \mathrm{e}^{u^{-} \cdot(u-b)}}{\mathrm{e}^{-u^{+} \cdot(u-b)}-\mathrm{e}^{u^{-} \cdot(u-b)}} .
$$

Thus,

$$
\begin{aligned}
& \mathrm{E}\left(\mathrm{e}^{-\gamma \tau(l, u)} ; X_{\tau(l, u)}=u \mid X_{0}=b\right) \\
& =\frac{u^{+}+u^{-}}{\mathrm{e}^{-u^{+} \cdot(u-b)}-\mathrm{e}^{u^{-} \cdot(u-b)}} \\
& \quad \times\left(\frac{u^{+} \mathrm{e}^{-u^{+} \cdot(b-l)}+u^{-} \mathrm{e}^{u^{-} \cdot(b-l)}}{\mathrm{e}^{-u^{+} \cdot(b-l)}-\mathrm{e}^{u^{-} \cdot(b-l)}}+\frac{u^{-} \mathrm{e}^{-u^{+} \cdot(u-b)}+u^{+} \mathrm{e}^{u^{-} \cdot(u-b)}}{\mathrm{e}^{-u^{+} \cdot(u-b)}-\mathrm{e}^{u^{-} \cdot(u-b)}}\right)^{-1} .
\end{aligned}
$$

Extending the fractions by $\left(\mathrm{e}^{-u^{+} \cdot(b-l)}-\mathrm{e}^{u^{-} \cdot(b-l)}\right)\left(\mathrm{e}^{-u^{+} \cdot(u-b)}-\mathrm{e}^{u^{-} \cdot(u-b)}\right)$ yields

$$
\begin{aligned}
& \mathrm{E}\left(\mathrm{e}^{-\gamma \tau(l, u)} ; X_{\tau(l, u)}=u \mid X_{0}=b\right) \\
&=\left(u^{+}+u^{-}\right)\left(\mathrm{e}^{-u^{+} \cdot(b-l)}-\mathrm{e}^{u^{-} \cdot(b-l)}\right) \\
& \quad \times\left(\left(u^{+} \mathrm{e}^{-u^{+} \cdot(b-l)}+u^{-} \mathrm{e}^{u^{-} \cdot(b-l)}\right)\left(\mathrm{e}^{-u^{+} \cdot(u-b)}-\mathrm{e}^{u^{-} \cdot(u-b)}\right)\right. \\
&\left.\quad \quad+\left(u^{-} \mathrm{e}^{-u^{+} \cdot(u-b)}+u^{+} \mathrm{e}^{u^{-} \cdot(u-b)}\right)\left(\mathrm{e}^{-u^{+} \cdot(b-l)}-\mathrm{e}^{u^{-} \cdot(b-l)}\right)\right)^{-1} \\
&=\left(u^{+}+u^{-}\right)\left(\mathrm{e}^{-u^{+} \cdot(b-l)}-\mathrm{e}^{u^{-} \cdot(b-l)}\right) \\
& \times\left(u^{+} \mathrm{e}^{-u^{+} \cdot(u-l)}-u^{-} \mathrm{e}^{u^{-} \cdot(u-l)}+u^{-} \mathrm{e}^{-u^{+} \cdot(u-l)}-u^{+} \mathrm{e}^{u^{-} \cdot(u-l)}\right)^{-1} \\
&= \frac{\mathrm{e}^{-u^{+} \cdot(b-l)}-\mathrm{e}^{u^{-} \cdot(b-l)}}{\mathrm{e}^{-u^{+} \cdot(u-l)}-\mathrm{e}^{u^{-} \cdot(u-l)}},
\end{aligned}
$$

which is the classical result; cf. Equation (2.17) of [9].

\subsection{Occupation times before a first passage}

Choose some $b<u \in \mathbb{R}$. Define the column vectors $\zeta_{k}(u):=\left(\zeta_{k j}(u): j \in E\right)$ for $k \in\{1,2\}$ by

$$
\zeta_{1, j}(u):=\int_{0}^{\tau(u)} \mathbf{1}_{\left\{X_{t}<b, J_{t}=j\right\}} \mathrm{d} t \quad \text { and } \quad \zeta_{2, j}(u):=\int_{0}^{\tau(u)} \mathbf{1}_{\left\{X_{t}>b, J_{t}=j\right\}} \mathrm{d} t
$$

for $j \in E$. Furthermore, choose any exit rate vectors $\boldsymbol{r}_{k}=\left(r_{k j}: j \in E\right)$ for $k \in\{1,2\}$. We shall derive an expression for

$$
E^{+}(u \mid a):=\mathrm{E}\left(\mathrm{e}^{-r_{1} \zeta_{1}(u)} \mathrm{e}^{-r_{2} \zeta_{2}(u)} \mid X_{0}=a\right),
$$

where $a<u$, thus providing the joint Laplace transform of the occupation times $\zeta_{k j}(u)$. There are three cases. If $a<u<b$ then $\sum_{j \in E} \zeta_{1, j}(u)=\tau(u)$ and, thus,

$$
E^{+}(u \mid a)=\mathrm{E}\left(\exp \left[-\int_{0}^{\tau(u)} \boldsymbol{r}_{1} e_{J_{s}} \mathrm{~d} s\right] \mid X_{0}=a\right)
$$


which has been determined in (2). If $a<b<u$ then

$$
E^{+}(u \mid a)=\mathrm{E}\left(\exp \left[-\int_{0}^{\tau(b)} \boldsymbol{r}_{1} e_{J_{s}} \mathrm{~d} s\right] \mid X_{0}=a\right) E^{+}(u \mid b)
$$

where again the first factor is known via (2). Finally, if $b<a<u$ then

$$
E^{+}(u \mid a)=\Psi_{r_{2}}^{+}(b, u \mid a)+\Psi_{r_{2}}^{-}(b, u \mid a) E^{+}(u \mid b),
$$

where the terms $\Psi_{r_{2}}^{+}$and $\Psi_{r_{2}}^{-}$are given in (7) and (8). Thus, it suffices to determine $E^{+}(u \mid b)$ for $b<u$. Clearly, $E_{(\cdot, d)}^{+}(u \mid b)=\mathbf{0}$, since $u$ cannot be passed from below in a descending phase. We further find the relation

$$
E_{(d, a)}^{+}(u \mid b)=\mathrm{E}_{(d, a)}\left(\mathrm{e}^{-r_{1} \zeta_{1}(b)} \mid X_{0}=b\right) E_{(a, a)}^{+}(u \mid b),
$$

where it remains to determine $E_{(a, a)}^{+}(u \mid b)$. Conditioning on the number $n$ of possible returns to the level $b$ in a strictly ascending phase $i \in E_{s}$ before passing the level $u$, we observe that

$$
\begin{aligned}
E_{(s, a)}^{+}(u \mid b) & =\sum_{n=0}^{\infty}\left(\Psi_{\boldsymbol{r}_{2}}^{-} C^{+}\left(\boldsymbol{r}_{1}\right)\right)_{(s, s)}^{n}\left(\left(\Psi_{\boldsymbol{r}_{2}}^{+}\right)_{(s, a)}+\left(\Psi_{\boldsymbol{r}_{2}}^{-} C^{+}\left(\boldsymbol{r}_{1}\right)\right)_{(s, \sigma)} E_{(\sigma, a)}^{+}(u \mid b)\right) \\
& =\left(I_{s}-\left(\Psi_{\boldsymbol{r}_{2}}^{-} C^{+}\left(\boldsymbol{r}_{1}\right)\right)_{(s, s)}\right)^{-1}\left(\left(\Psi_{\boldsymbol{r}_{2}}^{+}\right)_{(s, a)}+\left(\Psi_{\boldsymbol{r}_{2}}^{-} C^{+}\left(\boldsymbol{r}_{1}\right)\right)_{(s, \sigma)} E_{(\sigma, a)}^{+}(u \mid b)\right),
\end{aligned}
$$

where $I_{S}$ denotes the identity matrix on $E_{S}, \Psi_{\boldsymbol{r}_{2}}^{ \pm}=\Psi_{\boldsymbol{r}_{2}}^{ \pm}(b, u \mid b)$, and $C^{+}\left(\boldsymbol{r}_{1}\right)$ is given in (6). We have thus reduced the problem to the determination of $E_{(\sigma, a)}^{+}(u \mid b)$. This can be obtained as the limit $E_{(\sigma, a)}^{+}(u \mid b)=\lim _{l \rightarrow-\infty} E_{(\sigma, a)}^{+}(l, u \mid b)$.

Corollary 1. Write $U_{k}^{ \pm}:=U^{ \pm}\left(\boldsymbol{r}_{k}\right)$ for $k \in\{1,2\}$, and assume that $\left\|\boldsymbol{r}_{1}\right\|>0$. Then

$$
\begin{aligned}
E_{(\sigma, a)}^{+}(u \mid b)= & \mathrm{E}_{(\sigma, a)}\left(\mathrm{e}^{-\boldsymbol{r}_{1} \zeta_{1}(u)} \mathrm{e}^{-\boldsymbol{r}_{2} \zeta_{2}(u)} \mid X_{0}=b\right) \\
= & 2\left(\left(D_{2} C_{1}^{+}\right)_{(\sigma, \sigma)}+\left(2 U_{1}^{+}\right)_{(\sigma, \sigma)}\right)^{-1} \\
& \times\left(\mathbf{0}_{(\sigma, s)}, I_{\sigma}\right)\left(U_{2}^{+}+C_{2}^{-} U_{2}^{-} C_{2}^{+}\right)\left(\mathrm{e}^{-U_{2}^{+} \cdot(u-b)}-C_{2}^{-} \mathrm{e}^{U_{2}^{-} \cdot(u-b)} C_{2}^{+}\right)^{-1}
\end{aligned}
$$

for $b<u$, where the matrix $D_{2}$ is given in Lemma 2 .

Proof. Looking at the formula in Theorem 1 we find that $D_{1}$ depends only on $l$. For $l \rightarrow-\infty$, we obtain, from Lemma 1 ,

$$
\begin{aligned}
& \lim _{l \rightarrow-\infty} D_{1}= \lim _{l \rightarrow-\infty} 2\left(\mathbf{0}_{(\sigma, s)}, I_{\sigma}\right)\left(U_{1}^{+} \mathrm{e}^{-U_{1}^{+} \cdot(b-l)}+C_{1}^{-} U_{1}^{-} \mathrm{e}^{U_{1}^{-} \cdot(b-l)} C_{1}^{+}\right) \\
& \times\left(\mathrm{e}^{-U_{1}^{+} \cdot(b-l)}-C_{1}^{-} \mathrm{e}^{U_{1}^{-} \cdot(b-l)} C_{1}^{+}\right)^{-1} \\
&=\lim _{l \rightarrow-\infty} 2\left(\mathbf{0}_{(\sigma, s)}, I_{\sigma}\right)\left(U_{1}^{+}+C_{1}^{-} U_{1}^{-} \mathrm{e}^{U_{1}^{-} \cdot(b-l)} C_{1}^{+} \mathrm{e}^{U_{1}^{+} \cdot(b-l)}\right) \\
& \times\left(I_{a}-C_{1}^{-} \mathrm{e}^{U_{1}^{-} \cdot(b-l)} C_{1}^{+} \mathrm{e}^{U_{1}^{+} \cdot(b-l)}\right)^{-1} \\
&=2\left(\mathbf{0}_{(\sigma, s)}, I_{\sigma}\right) U_{1}^{+},
\end{aligned}
$$

since $\left\|\boldsymbol{r}_{1}\right\|>0$ implies that $U_{1}^{+}$is a strict subgenerator matrix and $\lim _{l \rightarrow-\infty} \mathrm{e}^{U_{1}^{+} \cdot(b-l)}=\mathbf{0}$. 
Example 6. For the compound Poisson risk model with exponential claims, the phase space is $E=E_{s} \cup E_{d}$, i.e. $E_{\sigma}=\varnothing$. Thus, $E_{(a, a)}^{+}(u \mid b)=E_{(s, s)}^{+}(u \mid b)$, for which the formula before Corollary 1 yields

$$
E_{(s, s)}^{+}(u \mid b)=\left(1-e_{1}^{\prime} \Psi_{\boldsymbol{r}_{2}}^{-} A^{+}\left(\boldsymbol{r}_{1}\right)\right)^{-1} e_{1}^{\prime} \Psi_{\boldsymbol{r}_{2}}^{+},
$$

as $C^{+}\left(\boldsymbol{r}_{1}\right)=A^{+}\left(\boldsymbol{r}_{1}\right)$ and $\Psi_{\boldsymbol{r}_{2}}^{ \pm}$are simply column vectors on $E$. Setting $\boldsymbol{r}_{1}=(0, \gamma), \boldsymbol{r}_{2}=\mathbf{0}$, and $b=0$, we obtain the Laplace transform (with argument $\gamma$ ) of the time spent above the initial risk reserve $u$ before ruin. The values for $\boldsymbol{r}_{2}$ yield $A_{2}^{+}=\lambda /(c \beta)$ and $U_{2}^{+}=\lambda / c-\beta$ as well as $A_{2}^{-}=1$ and $U_{2}^{-}=0$. Hence,

$$
e_{1}^{\prime} \Psi_{r_{2}}^{-}=\frac{1-\mathrm{e}^{-(\beta-\lambda / c) \cdot(u-b)}}{1-\lambda \mathrm{e}^{-(\beta-\lambda / c) \cdot(u-b)} / c \beta}
$$

and

$$
e_{1}^{\prime} \Psi_{r_{2}}^{+}=\frac{1-\lambda /(c \beta)}{1-\lambda \mathrm{e}^{-(\beta-\lambda / c) \cdot(u-b)} /(c \beta)} \mathrm{e}^{-(\beta-\lambda / c) \cdot(u-b)},
$$

while the values for $\boldsymbol{r}_{1}$ yield

$$
A^{+}\left(\boldsymbol{r}_{1}\right)=\frac{1}{2 c \beta}\left(\lambda+\gamma+c \beta-\sqrt{(\lambda+\gamma+c \beta)^{2}-4 \lambda c \beta}\right) .
$$

\section{Occupation times in more than two intervals}

We now consider a finite number of thresholds $b_{1}<\cdots<b_{N}$. We can determine the occupation times of $(\mathcal{X}, \mathcal{g})$ before a first passage or an exit from an interval in the following way. Fix the respective rate exit vector $\boldsymbol{r}_{k}$ for the (open) intervals $I_{k}, k \in\{1, \ldots, N+1\}$, resulting from $b_{1}<\cdots<b_{N}$. Define $\tau^{+}(x):=\inf \left\{t \geq 0: X_{t}>x\right\}$ and the column vectors $\zeta_{k}^{+}(u):=\left(\zeta_{k j}^{+}(u): j \in E\right), k \in\{1, \ldots, N+1\}$, by

$$
\zeta_{k j}^{+}(u):=\int_{0}^{\tau^{+}(u)} \mathbf{1}_{\left\{X_{t} \in I_{k}, J_{t}=j\right\}} \mathrm{d} t
$$

for $j \in E$. As before, we shall write

$$
E^{+}(u \mid a):=\mathrm{E}\left(\exp \left[-\sum_{k=1}^{N+1} \boldsymbol{r}_{k} \zeta_{k}^{+}(u)\right] \mid X_{0}=a\right)
$$

for $a<u$. Similarly, define $\tau^{-}(x):=\inf \left\{t \geq 0: X_{t}<x\right\}$ and the column vectors $\zeta_{k}^{-}(u):=$ $\left(\zeta_{k j}^{-}(u): j \in E\right), k \in\{1, \ldots, N+1\}$, by

$$
\zeta_{k j}^{-}(u):=\int_{0}^{\tau^{-}(u)} \mathbf{1}_{\left\{X_{t} \in I_{k}, J_{t}=j\right\}} \mathrm{d} t
$$

for $j \in E$. We shall write

$$
E^{-}(l \mid a):=\mathrm{E}\left(\exp \left[-\sum_{k=1}^{N+1} \boldsymbol{r}_{k} \zeta_{k}^{-}(l)\right] \mid X_{0}=a\right)
$$


for $l<a$. Define the column vectors $\zeta_{k}(l, u):=\left(\zeta_{k j}(l, u): j \in E\right), k \in\{1, \ldots, N+1\}$, by

$$
\zeta_{k j}(l, u):=\int_{0}^{\tau(l, u)} \mathbf{1}_{\left\{X_{t} \in I_{k}, J_{t}=j\right\}} \mathrm{d} t
$$

for $j \in E$. We write

$$
E^{+}(l, u \mid a):=\mathrm{E}\left(\exp \left[-\sum_{k=1}^{N+1} \boldsymbol{r}_{k} \zeta_{k}(l, u)\right] ; X_{\tau(l, u)}=u \mid X_{0}=a\right)
$$

and

$$
E^{-}(l, u \mid a):=\mathrm{E}\left(\exp \left[-\sum_{k=1}^{N+1} \boldsymbol{r}_{k} \zeta_{k}(l, u)\right] ; X_{\tau(l, u)}=l \mid X_{0}=a\right)
$$

for $l<a<u$. For $N=1$, the matrices $E^{+}(u \mid a)$ and $E^{+}(l, u \mid a)$ have been determined in Sections 4.2 and 4.1, respectively. The matrices $E^{-}(l \mid a)$ and $E^{-}(l, u \mid a)$ are determined in the same way after reflection at the initial level $a$, i.e. interchanging $A^{+}$and $U^{+}$with $A^{-}$and $U^{-}$; cf. the relation between (7) and (8).

\subsection{Occupation times before a first passage}

We seek a computational scheme for $E^{+}(u \mid a)$ where $a<u$. If $u \leq b_{2}$ then the solution is given by the results in Section 4.2 with $b=b_{1}$. For $u>b_{2}$, let $k:=\max \left\{n \geq 2: b_{n}<u\right\}$. Path continuity yields, for $a \leq b_{k}<u$,

$$
E^{+}(u \mid a)=E^{+}\left(b_{k} \mid a\right) E^{+}\left(u \mid b_{k}\right)
$$

where

$$
E^{+}\left(u \mid b_{k}\right)=E^{+}\left(b_{k-1}, u \mid b_{k}\right)+E^{-}\left(b_{k-1}, u \mid b_{k}\right) E^{+}\left(b_{k} \mid b_{k-1}\right) E^{+}\left(u \mid b_{k}\right),
$$

which implies that

$$
E^{+}\left(u \mid b_{k}\right)=\left(I-E^{-}\left(b_{k-1}, u \mid b_{k}\right) E^{+}\left(b_{k} \mid b_{k-1}\right)\right)^{-1} E^{+}\left(b_{k-1}, u \mid b_{k}\right) .
$$

In the case $b_{k}<a<u$ we obtain

$$
E^{+}(u \mid a)=\Psi_{k+1}^{+}\left(b_{k}, u \mid a\right)+\Psi_{k+1}^{-}\left(b_{k}, u \mid a\right) E^{+}\left(u \mid b_{k}\right),
$$

where $\Psi_{k+1}^{+}$and $\Psi_{k+1}^{-}$denote the two-sided exit matrices as defined in (7) and (8) with parameters taken from the $(k+1)$ th regime. Since the matrices $E^{+}\left(b_{k-1}, u \mid b_{k}\right)$ and $E^{-}\left(b_{k-1}, u \mid b_{k}\right)$ have been determined in Section 4.1, this provides a recursion scheme for $E^{+}(u \mid a)$.

\subsection{Occupation times before an exit from an interval}

We shall determine $E^{+}(l, u \mid a)$ with $l<a<u$. First note that the problem can be reduced to the results obtained in Section 5.1 by exploiting the probabilistic interpretation at the beginning of Section 3. This yields

$$
E^{+}(l, u \mid a)=\left(E^{+}(u \mid a)-E^{-}(l \mid a) E^{+}(u \mid l)\right)\left(I-E^{-}(l \mid u) E^{+}(u \mid l)\right)^{-1} .
$$

We further wish to provide a recursion that involves only matrices of the form $E^{ \pm}(x, y \mid z)$. For $h:=\min \left\{n \geq 1: b_{n}>l\right\}$, the matrix $E^{+}\left(l, b_{h+1} \mid b_{h}\right)$ has been determined in Section 4.1. 
Define $k:=\max \left\{n \geq 1: b_{n}<u\right\}$. If $k=h$ then $E^{+}(l, u \mid a)$ is given by the results in Section 4.1. Thus, assume that $k>h \geq 1$. We obtain, by path continuity,

$$
E^{+}(l, u \mid a)=E^{+}\left(l, b_{k} \mid a\right) E^{+}\left(l, u \mid b_{k}\right),
$$

where

$$
E^{+}\left(l, u \mid b_{k}\right)=E^{+}\left(b_{k-1}, u \mid b_{k}\right)+E^{-}\left(b_{k-1}, u \mid b_{k}\right) E^{+}\left(l, b_{k} \mid b_{k-1}\right) E^{+}\left(l, u \mid b_{k}\right) .
$$

This yields

$$
E^{+}\left(l, u \mid b_{k}\right)=\left(I-E^{-}\left(b_{k-1}, u \mid b_{k}\right) E^{+}\left(l, b_{k} \mid b_{k-1}\right)\right)^{-1} E^{+}\left(b_{k-1}, u \mid b_{k}\right) .
$$

Since the matrices $E^{+}\left(b_{k-1}, u \mid b_{k}\right)$ and $E^{-}\left(b_{k-1}, u \mid b_{k}\right)$ have been determined in Section 4.1, this provides a recursion scheme for $E^{+}(l, u \mid a)$.

\section{Appendix A}

In this appendix the lemmata that have been used in the proof of Theorem 1 are collected. Recall the abbreviations $\Psi_{1}^{+}(\varepsilon)$ for the $\left(\left(E_{\sigma} \cup E_{d}\right) \times E_{a}\right)$-block of $\Psi_{r_{1}}^{+}(l, b+\varepsilon \mid b-\varepsilon), \Psi_{2}^{-}(\varepsilon)$ for the $\left(E_{a} \times\left(E_{\sigma} \cup E_{d}\right)\right)$-block of $\Psi_{\boldsymbol{r}_{2}}^{-}(b-\varepsilon, u \mid b+\varepsilon)$, and $\Psi_{2}^{+}(\varepsilon)$ for the $\left(E_{a} \times E_{a}\right)$-block of $\Psi_{\boldsymbol{r}_{2}}^{+}(b-\varepsilon, u \mid b+\varepsilon)$. Furthermore, let $\mathbf{0}_{(\sigma, d)}$ and $\mathbf{0}_{(\sigma, s)}$ denote the zero matrices on $E_{\sigma} \times E_{d}$ and on $E_{\sigma} \times E_{s}$, respectively.

Lemma 1. For $l<b$,

$$
\begin{aligned}
D_{1}:= & \left.\left(I_{\sigma}, \mathbf{0}_{(\sigma, d)}\right) \frac{\mathrm{d}}{\mathrm{d} \varepsilon} \Psi_{1}^{+}(\varepsilon)\right|_{\varepsilon=0} \\
= & 2\left(\mathbf{0}_{(\sigma, s)}, I_{\sigma}\right)\left(U_{1}^{+} \mathrm{e}^{-U_{1}^{+} \cdot(b-l)}+C_{1}^{-} U_{1}^{-} \mathrm{e}^{U_{1}^{-} \cdot(b-l)} C_{1}^{+}\right) \\
& \times\left(\mathrm{e}^{-U_{1}^{+} \cdot(b-l)}-C_{1}^{-} \mathrm{e}^{U_{1}^{-} \cdot(b-l)} C_{1}^{+}\right)^{-1} .
\end{aligned}
$$

Proof. According to (7),

$$
\begin{aligned}
\left(I_{\sigma}, \mathbf{0}_{(\sigma, d)}\right) \Psi_{1}^{+}(\varepsilon)= & \left(I_{\sigma}, \mathbf{0}_{(\sigma, d)}\right)\left(C_{1}^{+} \mathrm{e}^{U_{1}^{+} \cdot(2 \varepsilon)}-\mathrm{e}^{U_{1}^{-} \cdot(b-l-\varepsilon)} C_{1}^{+} \mathrm{e}^{U_{1}^{+} \cdot(b-l+\varepsilon)}\right) \\
& \times\left(I_{a}-C_{1}^{-} \mathrm{e}^{U_{1}^{-} \cdot(b-l+\varepsilon)} C_{1}^{+} \mathrm{e}^{U_{1}^{+} \cdot(b-l+\varepsilon)}\right)^{-1} \\
= & \left(\mathbf{0}_{(\sigma, s)}, I_{\sigma}\right)\left(\mathrm{e}^{U_{1}^{+} \cdot(2 \varepsilon)}-C_{1}^{-} \mathrm{e}^{U_{1}^{-} \cdot(b-l-\varepsilon)} C_{1}^{+} \mathrm{e}^{U_{1}^{+} \cdot(b-l+\varepsilon)}\right) \\
& \times\left(I_{a}-C_{1}^{-} \mathrm{e}^{U_{1}^{-} \cdot(b-l+\varepsilon)} C_{1}^{+} \mathrm{e}^{U_{1}^{+} \cdot(b-l+\varepsilon)}\right)^{-1} \\
= & \left(\mathbf{0}_{(\sigma, s)}, I_{\sigma}\right)\left(\mathrm{e}^{-U_{1}^{+} \cdot(b-l-\varepsilon)}-C_{1}^{-} \mathrm{e}^{U_{1}^{-} \cdot(b-l-\varepsilon)} C_{1}^{+}\right) \\
& \times\left(\mathrm{e}^{-U_{1}^{+} \cdot(b-l+\varepsilon)}-C_{1}^{-} \mathrm{e}^{U_{1}^{-} \cdot(b-l+\varepsilon)} C_{1}^{+}\right)^{-1}
\end{aligned}
$$

Letting

$$
F(\varepsilon):=\mathrm{e}^{-U_{1}^{+} \cdot(b-l-\varepsilon)}-C_{1}^{-} \mathrm{e}^{U_{1}^{-} \cdot(b-l-\varepsilon)} C_{1}^{+}
$$

and

$$
G(\varepsilon):=\mathrm{e}^{-U_{1}^{+} \cdot(b-l+\varepsilon)}-C_{1}^{-} \mathrm{e}^{U_{1}^{-} \cdot(b-l+\varepsilon)} C_{1}^{+},
$$

and applying the formal rules of derivation for functions of a real variable (see [4, Sections I.1.34]), we obtain

$$
D_{1}=\left(\mathbf{0}_{(\sigma, s)}, I_{\sigma}\right)\left(F^{\prime}(0) G(0)^{-1}-F(0) G(0)^{-1} G^{\prime}(0) G(0)^{-1}\right),
$$


where $F(0)=G(0)=\mathrm{e}^{-U_{1}^{+} \cdot(b-l)}-C_{1}^{-} \mathrm{e}^{U_{1}^{-} \cdot(b-l)} C_{1}^{+}$and

$$
F^{\prime}(0)=U_{1}^{+} \mathrm{e}^{-U_{1}^{+} \cdot(b-l)}+C_{1}^{-} U_{1}^{-} \mathrm{e}^{U_{1}^{-} \cdot(b-l)} C_{1}^{+}=-G^{\prime}(0) .
$$

This completes the proof.

Lemma 2. For $b<u$,

$$
\begin{aligned}
D_{2}:= & \left.\left(\mathbf{0}_{(\sigma, s)}, I_{\sigma}\right) \frac{\mathrm{d}}{\mathrm{d} \varepsilon} \Psi_{2}^{-}(\varepsilon)\right|_{\varepsilon=0} \\
= & 2\left(I_{\sigma}, \mathbf{0}_{(\sigma, d)}\right)\left(U_{2}^{-} \mathrm{e}^{-U_{2}^{-} \cdot(u-b)}+C_{2}^{+} U_{2}^{+} \mathrm{e}^{U_{2}^{+} \cdot(u-b)} C_{2}^{-}\right) \\
& \times\left(\mathrm{e}^{-U_{2}^{-} \cdot(u-b)}-C_{2}^{+} \mathrm{e}^{U_{2}^{+} \cdot(u-b)} C_{2}^{-}\right)^{-1} .
\end{aligned}
$$

Proof. According to (8),

$$
\begin{aligned}
\left(\mathbf{0}_{(\sigma, s)}, I_{\sigma}\right) \Psi_{2}^{-}(\varepsilon)= & \left(\mathbf{0}_{(\sigma, s)}, I_{\sigma}\right)\left(C_{2}^{-} \mathrm{e}^{U_{2}^{-} 2 \varepsilon}-\mathrm{e}^{U_{2}^{+} \cdot(u-b-\varepsilon)} C_{2}^{-} \mathrm{e}^{U_{2}^{-} \cdot(u-b+\varepsilon)}\right) \\
& \times\left(I-C_{2}^{+} \mathrm{e}^{U_{2}^{+} \cdot(u-b+\varepsilon)} C_{2}^{-} \mathrm{e}^{U_{2}^{-} \cdot(u-b+\varepsilon)}\right)^{-1} \\
= & \left(I_{\sigma}, \mathbf{0}_{(\sigma, d)}\right)\left(\mathrm{e}^{U_{2}^{-} 2 \varepsilon}-C_{2}^{+} \mathrm{e}^{U_{2}^{+} \cdot(u-b-\varepsilon)} C_{2}^{-} \mathrm{e}^{U_{2}^{-} \cdot(u-b+\varepsilon)}\right) \\
& \times\left(I-C_{2}^{+} \mathrm{e}^{U_{2}^{+} \cdot(u-b+\varepsilon)} C_{2}^{-} \mathrm{e}^{U_{2}^{-} \cdot(u-b+\varepsilon)}\right)^{-1} \\
= & \left(I_{\sigma}, \mathbf{0}_{(\sigma, d)}\right)\left(\mathrm{e}^{-U_{2}^{-} \cdot(u-b-\varepsilon)}-C_{2}^{+} \mathrm{e}^{U_{2}^{+} \cdot(u-b-\varepsilon)} C_{2}^{-}\right) \\
& \times\left(\mathrm{e}^{-U_{2}^{-} \cdot(u-b+\varepsilon)}-C_{2}^{+} \mathrm{e}^{U_{2}^{+} \cdot(u-b+\varepsilon)} C_{2}^{-}\right)^{-1} .
\end{aligned}
$$

Let

$$
F(\varepsilon):=\mathrm{e}^{-U_{2}^{-} \cdot(u-b-\varepsilon)}-C_{2}^{+} \mathrm{e}^{U_{2}^{+} \cdot(u-b-\varepsilon)} C_{2}^{-}
$$

and

$$
G(\varepsilon):=\mathrm{e}^{-U_{2}^{-} \cdot(u-b+\varepsilon)}-C_{2}^{+} \mathrm{e}^{U_{2}^{+} \cdot(u-b+\varepsilon)} C_{2}^{-},
$$

where $F(0)=G(0)=\mathrm{e}^{-U_{2}^{-} \cdot(u-b)}-C_{2}^{+} \mathrm{e}^{U_{2}^{+} \cdot(u-b)} C_{2}^{-}$and

$$
F^{\prime}(0)=U_{2}^{-} \mathrm{e}^{-U_{2}^{-} \cdot(u-b)}+C_{2}^{+} U_{2}^{+} \mathrm{e}^{U_{2}^{+} \cdot(u-b)} C_{2}^{-}=-G^{\prime}(0) .
$$

Hence,

$$
\begin{aligned}
D_{2} & =\left(I_{\sigma}, \mathbf{0}_{(\sigma, d)}\right)\left(F^{\prime}(0) G(0)^{-1}-F(0) G(0)^{-1} G^{\prime}(0) G(0)^{-1}\right) \\
& =2\left(I_{\sigma}, \mathbf{0}_{(\sigma, d)}\right) F^{\prime}(0) G(0)^{-1},
\end{aligned}
$$

completing the proof.

Lemma 3. For $b<u$,

$$
\begin{aligned}
D_{3} & :=\left.\left(\mathbf{0}_{(\sigma, s)}, I_{\sigma}\right) \frac{\mathrm{d}}{\mathrm{d} \varepsilon} \Psi_{2}^{+}(\varepsilon)\right|_{\varepsilon=0} \\
& =-2\left(\mathbf{0}_{(\sigma, s)}, I_{\sigma}\right)\left(U_{2}^{+}+C_{2}^{-} U_{2}^{-} C_{2}^{+}\right)\left(\mathrm{e}^{-U_{2}^{+} \cdot(u-b)}-C_{2}^{-} \mathrm{e}^{U_{2}^{-} \cdot(u-b)} C_{2}^{+}\right)^{-1} .
\end{aligned}
$$


Proof. According to (7),

$$
\begin{aligned}
\left(\mathbf{0}_{(\sigma, s)}, I_{\sigma}\right) \Psi_{2}^{+}(\varepsilon)= & \left(\mathbf{0}_{(\sigma, s)}, I_{\sigma}\right)\left(\mathrm{e}^{U_{2}^{+} \cdot(u-b-\varepsilon)}-C_{2}^{-} \mathrm{e}^{U_{2}^{-} 2 \varepsilon} C_{2}^{+} \mathrm{e}^{U_{2}^{+} \cdot(u-b+\varepsilon)}\right) \\
& \times\left(I_{a}-C_{2}^{-} \mathrm{e}^{U_{2}^{-} \cdot(u-b+\varepsilon)} C_{2}^{+} \mathrm{e}^{U_{2}^{+} \cdot(u-b+\varepsilon)}\right)^{-1} \\
= & \left(\mathbf{0}_{(\sigma, s)}, I_{\sigma}\right)\left(\mathrm{e}^{-U_{2}^{+} \cdot 2 \varepsilon}-C_{2}^{-} \mathrm{e}^{U_{2}^{-} 2 \varepsilon} C_{2}^{+}\right) \\
& \times\left(\mathrm{e}^{-U_{2}^{+} \cdot(u-b+\varepsilon)}-C_{2}^{-} \mathrm{e}^{U_{2}^{-} \cdot(u-b+\varepsilon)} C_{2}^{+}\right)^{-1} .
\end{aligned}
$$

\section{Letting}

$$
F(\varepsilon)=\mathrm{e}^{-2 U_{2}^{+} \varepsilon}-C_{2}^{-} \mathrm{e}^{2 U_{2}^{-} \varepsilon} C_{2}^{+} \quad \text { and } \quad G(\varepsilon)=\mathrm{e}^{-U_{2}^{+} \cdot(u-b+\varepsilon)}-C_{2}^{-} \mathrm{e}^{U_{2}^{-} \cdot(u-b+\varepsilon)} C_{2}^{+},
$$

we obtain $F^{\prime}(0)=-2\left(U_{2}^{+}+C_{2}^{-} U_{2}^{-} C_{2}^{+}\right),\left(\mathbf{0}_{(\sigma, s)}, I_{\sigma}\right) F(0)=\mathbf{0}_{(\sigma, a)}$, and $G(0)=\mathrm{e}^{-U_{2}^{+} \cdot(u-b)}-$ $C_{2}^{-} \mathrm{e}^{U_{2}^{-} \cdot(u-b)} C_{2}^{+}$. Altogether, this yields

$$
\begin{aligned}
D_{3} & =\left(\mathbf{0}_{(\sigma, s)}, I_{\sigma}\right)\left(F^{\prime}(0) G(0)^{-1}-F(0) G(0)^{-1} G^{\prime}(0) G(0)^{-1}\right) \\
& =\left(\mathbf{0}_{(\sigma, s)}, I_{\sigma}\right) F^{\prime}(0) G(0)^{-1},
\end{aligned}
$$

completing the proof.

\section{References}

[1] Asmussen, S. (1995). Stationary distributions for fluid flow models with or without Brownian motion. Commun. Statist. Stoch. Models 11, 21-49.

[2] Asmussen, S. (2003). Applied Probability and Queues. Springer, New York.

[3] Asmussen, S., Avram, F. and Pistorius, M. R. (2004). Russian and American put options under exponential phase-type Lévy models. Stoch. Process. Appl. 109, 79-111.

[4] Bourbaki, N. (2004). Functions of a Real Variable. Springer, Berlin.

[5] Breuer, L. (2008). First passage times for Markov additive processes with positive jumps of phase type. J. Appl. Prob. 45, 779-799.

[6] Breuer, L. (2010). A quintuple law for Markov additive processes with phase-type jumps. J. Appl. Prob. 47, $441-458$.

[7] D’Auria, B., Ivanovs, J., Kella, O. and Mandjes, M. (2012). Two-sided reflection of Markov-modulated Brownian motion. Stoch. Models 28, 316-332.

[8] Gerber, H. U. and Shiu, E. S. W. (1998). On the time value of ruin. N. Amer. Actuarial J. 2, 48-78.

[9] Gerber, H. U. and Shiu, E. S. W. (2004). Optimal dividends: analysis with Brownian motion. N. Amer. Actuarial J. 8, 1-20.

[10] Ivanovs, J. (2010). Markov-modulated Brownian motion with two reflecting barriers. J. Appl. Prob. 47, 1034-1047.

[11] Ivanovs, J. (2011). One-sided Markov additive processes and related exit problems. Doctoral Thesis, Universiteit van Amsterdam.

[12] Ivanovs, J. AND Palmowski, Z. (2011). Occupation densities in solving exit problems for Markov additive processes and their reflections. Preprint. Available at http://arxiv.org/abs/1110.3811v1.

[13] Jiang, Z. and Pistorius, M. R. (2008). On perpetual American put valuation and first-passage in a regimeswitching model with jumps. Finance Stoch. 12, 331-355.

[14] Kyprianou, A. E. (2006). Introductory Lectures on Fluctuations of Lévy Processes with Applications. Springer, Berlin.

[15] Rogers, L. C. G. (1994). Fluid models in queueing theory and Wiener-Hopf factorization of Markov chains. Ann. Appl. Prob. 4, 390-413. 